\title{
Neural coding partially accounts for the relation between children's number line estimation and numerical comparison performance
}

\author{
Richard Prather \\ University of Maryland \\ Department of Human Development \& Quantitative Methodology
}

Numerical comparison is a primary measure of the acuity of children's approximate number system (ANS). ANS acuity is associated with key developmental outcomes such as symbolic number skill, standardized test scores and even employment outcomes(Halberda, Mazzocco, \& Feigenson, 2008; Parsons \& Bynner, 1997a). We examine the relation between children's performance on the numerical comparison task and the number line estimation task. It is important to characterize the relation between tasks in order to develop mathematics interventions that lead to transfer across tasks. We find that number line performance is significantly predicted by non-symbolic comparison performance for participants ranging in age from 5 to 8 years. We also evaluate, using a computational model, if the relation between the two tasks can be adequately explained based on known neural correlates of number perception. Data from humans and non-human primates characterizes neural activity corresponding to the perception of numerosities. Results of behavioral experimentation and computational modeling suggest that though neural coding of number predicts a correlation in participants' performance on the two tasks, it cannot account for all of the variability in the human data. This is interpreted as consistent with accounts of number line estimation in which number line estimation does not rely solely on participants' numerical perception.

\section{Introduction}

Children in school face the daunting task of learning a complex number system. Children must learn to manipulate numbers in increasingly sophisticated ways to stay on track in the formal education system. Understanding how different aspects of numerical cognition are related to one another within learners is an important goal for improving educational outcomes.

Numerical cognition research has focused on performance on a few model tasks, such as number comparison and number line estimation. Numerical comparison is used to measure number sense, learners' fluency with numbers. The number line estimation task measures number to space mapping ability, which has been shown to be important in numerical development (Gunderson, Ramirez, Beilock, \& Levine, 2012). It is unclear the degree to which performance on these two tasks relies on the same mechanisms. A central question is whether individual differences in performance across these tasks is primarily due to a shared cognitive mechanism.

\section{Study Motivation}

The current study addresses the questions, (1) does children's non-symbolic comparison skill predict performance on the symbolic number line task when accounting for age, (2) if so can a computational model of the neural coding of number account for the relation. Several cognitive representations have been used to characterize participants' behavior, such as the mental number 
line (e.g., Izard \& Dehaene, 2008) and neural tuning curves (Prather, 2012; 2014). We are interested specifically in the potential connection between non-symbolic comparison and symbolic number line for two reasons: First, the two tasks are amongst the most commonly used in assessing children's number skill. Secondly, the two tasks are dissimilar and yet may both rely on similar representations of number in terms of neural coding. Performance for both tasks improves with age and experience (Halberda \& Feigenson, 2008; Siegler \& Opfer, 2003). The question addressed here is whether the improvement is due to change in the same underlying mechanism or separate mechanisms that each happen to correlate with age.

We use a computational model to evaluate whether the relation between number line estimation and non-symbolic numerical comparison can be explained by neural coding of number. Prior work has shown that non-human primates' neural coding of number varies on trials where an incorrect answer is made. That is, neural coding "errors" correspond with behavioral errors (Nieder \& Dehaene, 2009; Nieder, 2005, 2012). The computational model used here relies on the correspondence of neural activity and behavior. The model calculates how simulated neural tuning curves may vary across task stimuli to estimate how behavior related to the task may also vary. Computational modeling results demonstrate that if neural coding as reported with nonhuman primates is assumed we can expect the pattern of errors seen with both the number line and non-symbolic number comparison task (Prather, 2012). We have also used this approach to predict novel behavioral phenomena for the non-symbolic numerical comparison task which were then confirmed though experiment (Prather, 2014).

While prior models have been used to account for behavior on both the number line estimation and non-symbolic number comparison tasks separately, the current study evaluates whether model parameters can account for individual participants' behavior on multiple tasks simultaneously (Prather, 2012; 2014). In this case, we attempt to model each participant individually, as opposed to modeling the average behavior for the entire group. This approach allows us to evaluate if the neural turning curve representation can account for the variation in participants' performance on both tasks. This will tell us more about why individual learners' performance on numerical comparison and number line estimation tasks are correlated. We hypothesize that the neural coding account, as instantiated by the computational model presented here, is sufficient to account for individual variation on the non-symbolic comparison and number line estimation tasks.

The computational model provides an algorithmic level of characterization for the relationship between learners' performance on the numerical comparison and number line estimation tasks (Marr, 1982). Algorithmic models are designed to make precise predictions that capture individual differences in behavior. The model evaluates how algorithmic parameters can be varied to fit each individual's data. This approach allows the model to account for more variation than a simple correlational model. For example, a group of participants may show a correlation of $\mathrm{R}^{2}=0.42$ between scores on the number-line estimation and numerical comparison tasks. Within that group two participants may perform equally well on the number-line task but differently on the numerical comparison task. A correlational approach describes the relationship between performances on the two tasks in terms of shared variance for the group. This sort of correlational analysis does not and cannot address why two participants may score identically on the number line task but differently on the numerical comparison task. A model of the underlying algorithms involved can address what aspects the cognitive mechanisms relevant to the two tasks could vary in a way that would lead to the difference observed between the participants The two participants may have similar representations of number via neural tuning curves but very 
different approaches to evidence accumulation in making a comparison between numerical values.

We present a behavioral experiment that includes measures of children's performance on symbolic number line estimation and non-symbolic numerical comparison. The behavioral experiment results are used input to a computational model. We demonstrate that the computational modeling approach has some limitations in accounting for individual differences in children's performance on both tasks. The combined results of the behavioral and computational experiments suggest that the model of neural tuning curves associated with number perception can account for some, but not all, of the individual differences in children's number line estimation and number comparison performance.

\section{What explains children's performance on the number line task?}

The number line estimation task involves mapping a numerical value onto a spatial representation. The typical format for the number line task is for participants to view a line with labeled endpoints, such as 1 and 100 (e.g., Opfer \& Siegler, 2007; Siegler \& Opfer, 2003). Participants are then asked to indicate where a particular number would go between those points by marking the line. Typically each trial is done with an empty number line and feedback is not given. The format of the number presentation, length of number line and method of response all vary across studies. Behavioral and developmental phenomena for the number line task are well documented (e.g., Cohen \& Blanc-Goldhammer, 2011; Geary, Hoard, Nugent, \& Byrd-Craven, 2008; Opfer \& Siegler, 2007; Siegler \& Opfer, 2003). For a given range of numbers (e.g. 1 - 100), young children (e.g., 4-5 years old) tend to produce overestimations on smaller numbers and underestimations for larger numbers, creating an estimation pattern that can be described as logarithmic. Older children (e.g., 7 - 8 years old) tend to be more accurate and eventually shift to a more linear pattern (Figure 1).

What accounts for the behavior on the number line task and how it changes with development? There are several accounts of the mechanisms that contribute to children's change in performance across development. One such account posits that children's cognitive development includes changes in mental number line representations (Opfer \& Siegler, 2007; Siegler \& Opfer, 2003). Younger children have a mental number line that results in logarithmic pattern of responses on the number line task. As children age they gain a linear mental number line and show improved performance.

Other accounts of number line task performance include proportional reasoning skill and measurement skill (e.g., Cohen \& Sarnecka, 2014; Reid, Baroody, \& Purpura, 2015). Evidence for the influence of proportional reasoning comes from findings suggesting that models using power functions fit account for more variance in children's estimates than models using logarithmic functions (Barth \& Paladino, 2011). Proportional reasoning may be a significant part of children's behavior on the number line task. Additionally recent work has noted performance depends on the type of numerical and spatial representations used in the task stimuli. For example, the use of an unbounded line changes participants' behavior (Cohen \& Sarnecka, 2014), as does the use of non-symbolic stimuli that are discrete or continuous (Sella, Berteletti, Lucangeli, \& Zorzi, 2015). The use of very large numerical values that the participant has less experience with also effects behavior on this task (Landy, Charlesworth, \& Ottmar, 2016).

\section{What explains children's number comparison performance?}


There is not a clear characterization of the cognitive mechanisms used to complete the numerical comparison task or why children's performance may change. Performance on the numerical comparison task is typically used as a measure of the numeracy construct. Numeracy in turn correlates with a variety of outcome measures related to mathematics. There are several accounts of the mechanisms involved in completing the numerical comparison task. One possibility is that variance in cognitive representations of number, such as mental number line (e.g., Izard \& Dehaene, 2008) or neural coding of number (Prather, 2014) explain performance. In both these cases the representations of the numbers to be compared is the primary variable that explains learners' performance on the task. Representations that are not precise are associated with poor performance on the task.

It is also possible that performance on the numerical comparison task is not best explained by representations of number but domain general skills involved in decision making. Other potential explanations include executive function (e.g., Cragg \& Gilmore, 2014), or domain general decision making skills (Holloway \& Ansari, 2008).

While these accounts are not necessarily mutually exclusive it is important that researchers accurately characterize the cognitive mechanisms involved in the task. An accurate characterization would allow for understanding of why performance on the task is associated with later outcomes. It would also allow for a characterization of the relation between performances on numerical tasks that conveys the causal mechanisms and not simply list associations. For example, why is there a correlation between numerical comparison and number line estimation? There are many differences in terms of what is required to complete either task. A significant correlation between learners' performances on the two tasks suggests a similarity in the underlying mechanism. However, a correlation does not explain what that mechanism may be or provide a means of predicting learners' performance on additional tasks. The mechanistic account described here, is constructed to do exactly those things.

\section{What explains the relation between numerical tasks?}

It is possible that completing the numerical comparison and number line estimation tasks involves a common mechanism or internal representation. Number line performance is correlated with non-symbolic number comparison skill (Fazio, Bailey, Thompson, \& Siegler, 2014; Laski \& Siegler, 2007), though in some cases the correlation disappears when age is controlled for (Sasanguie, Göbel, Moll, Smets, \& Reynvoet, 2013; Sasanguie \& Reynvoet, 2013). This correlation is important because non-symbolic number comparison is used to assess the acuity of learners' approximate number system, a mechanism for perceiving numerosity (e.g., Halberda \& Feigenson, 2008; Halberda, Mazzocco, \& Feigenson, 2008). Approximate number system acuity is linked to symbolic arithmetic skill, standardized test scores and other long-term outcomes (e.g., Libertus, Odic, \& Halberda, 2012; Parsons \& Bynner, 1997). Several recent meta-analyses suggest that there is a moderate effect of children's non-symbolic performance on math achievement (Chen \& $\mathrm{Li}$, 2014; Fazio et al., 2014; Schneider et al., 2016). Children's performance on symbolic number line estimation is correlated with symbolic numerical comparison (Laski \& Siegler, 2007) and nonsymbolic comparison (Fazio, Bailey, Thompson, \& Siegler, 2014). These results include bivariate correlations without accounting for the likely covariate of age. The current study attempts to disentangle the relation between task performance and age. Prior work suggest that when accounting for age there is not a relation between non-symbolic numerical and number line estimation (e.g., Sasanguie et al., 2013; Sasanguie \& Reynvoet, 2013). 
If the relationship between learners' performance on the numerical comparison and number line estimation tasks is due to a common mechanism or representation then more than a correlation coefficient is needed to characterize the mechanism or representation. The mechanism is characterized at an algorithmic level of analysis (Marr, 1982) using results from a behavioral experiment and computational model. The computational model includes a description of the potential algorithms involved in completing the number-line estimation and numerical comparison tasks. For example, in comparing numerical values behavior may depend on the learner's numerical representations, the accumulation of evidence for decision-making, or both. By focusing on an algorithmic level of analysis we can evaluate how learners' behavior may vary depending on variations in the mechanisms involved in completing the task. In the current study we expect the computational model to more accurately predict learners' performance on the number line task than could be done with simple correlations by including these other sources of variance in the model.

\section{The influence of neural coding on performance}

In the current study we evaluate whether the neural coding of number can account for individual differences in children's performance on number line estimation and numerical comparisons, as well as explaining the shared variance between these two tasks. If computational model of neural coding can account of the relation between the two tasks then we may be able to use the computational model to predict individual learners' performance on either task. A computational model that characterizes the underlying mechanisms involved in completing numerical tasks would be very useful for researchers. For example, a model can be used to evaluate behavioral data and in particular help to understand novel phenomena. The model can be used as an initial evaluation of possible transfer between tasks and the utility of interventions meant to improve performance. It could be used to make predictions about which learner's performance on difference tasks. It could also be used to make predictions about why some learners improve while others do not. Prior work has shown that computational models of neural coding can account for developmental change in number line estimation (Prather, 2012) and adult performance on the numerical comparison task (Prather, 2014). It is plausible that neural coding of number is a possible explanation for shared variance across number cognition tasks. However, prior work did not address whether a single model can account for participants behavior on both tasks simultaneously, leaving open the extent to which correlations between tasks might be explained by each task's reliance on numerical representations. Many accounts of numerical cognition rely on numerical representations to some degree. Determining how much variance numerical representations can account for in each task is a good first step in figuring out how to account for variation in learners' behavior on all numerical tasks.

The current study evaluates the hypothesis that variation in the neural coding of number can account for individual differences across the numerical comparison and number line estimation tasks. The study involves both behavioral data and computational models that are based on the patterns of neural activity reported and reports of how behavior can correlate with variations in activity (Prather, 2012; 2014). The neural coding account does not necessarily contradict prior accounts of number line estimation or numerical comparison. The neural coding account is not mutually exclusive with other accounts, such as the proportional judgment account of number line estimation (e.g., Slusser \& Barth, 2017). The neural coding account, as instantiated via computational modeling has the advantage of describing the mechanisms underlie behavior that allows for prediction of individual behavior across multiple tasks. 
The neural coding of numerical values may provide a mechanism for behavior and developmental changes on both the number line and numerical comparison tasks. Neurons that are sensitive to numerical perception show neural tuning curves: patterns of activity where the firing rate is greatest for a target number and relatively lower when for other numbers. Importantly these studies showed that behavioral errors on a numerical comparison task were correlated with neural coding "errors", in which a neuron responds to too wide a range of numbers (e.g., Nieder \& Dehaene, 2009; Nieder \& Merten, 2007). Prior research showed that the neural coding data can explain children's typical patterns of errors, as well as capture developmental change on the number line task (Prather, 2012). The model illustrates that the pattern of behavioral errors seen with children is a natural consequence of neural activation patterns.

\section{The current study}

If there is a common mechanism underlying the numerical comparison and number line tasks then we expect a significant statistical relation between participants' performance on the two tasks. We examine if performance on the number comparison task predicts children's number line task performance. The non-symbolic number comparison task is used as a measure of the numeracy construct for children as young as pre-school. Thus, our interest is if numerical comparison predicts symbolic number line performance, and not vice versa. The study is not designed to directly contradict or support specific prior studies. We construct a computational model that contributes to the characterization of the underlying mechanisms of individual differences in performance on numerical comparison and number line estimation tasks. One potential benefit of this approach is the future extrapolation of results to other numerical tasks not addressed here. A reproducible computational model of the representations and mechanisms involved in completing numerical tasks can be used to make predictions of individual learners' performance. Once we have a comprehensive model we will be better able to predict behavior predict how practice with a task transfers to a different task.

\section{Experiment 1}

\section{Method}

\section{Participants.}

Participants were children $(n=51)$ between ages of 5:5 and 8:11 (median 7:5). A small group $(n=4)$ of participants were not included in the analysis due to selection bias on the numerical comparison task, indicated by selection of one side (left or right) on $>80 \%$ of trials. Participants completed two tasks, a non-symbolic numerical comparison task and a symbolic number line estimation task. Task order was counter-balanced across participants. The experiment comprised of a laboratory session of approximately 30 minutes. Experimental design was approved through a research ethics board, the Indiana University Internal Review Board.

Non-symbolic numerical comparison. 
Each participant completed 51 trials of a forced choice comparison between pairs of sets of shapes presented simultaneously (see Appendix for list). The sets were comprised of squares that varied in area such that the two sets were matched in overall area, area of largest shape and area of smallest shape (Figure 2). Stimuli were presented to participants on laminated cards (8.5 x 11 inches). Participants were not given a time limit but were instructed to answer as quickly as they could. Stimuli were presented in a randomized order. Stimuli were comprised of 17 ratio differences from 1.03 to 1.18 , and 3 absolute value sizes for each ratio difference. For example for the ratio 1.04 the comparisons 25:26, 50:52 and 75:78 would be used. The location (left or right) of the larger value was counter balanced. Participants responded by pointing to which set they thought had a larger number of objects. Pilot testing indicated that this range would produce performance above chance and also well below ceiling. Our goal was to be able to observe variation in individuals' performance.

\section{Symbolic Number line estimation.}

Each participant completed 20 trials of a symbolic number line estimation task (e.g., Opfer \& Siegler, 2007). Participants were familiarized with a physical number line, $1 \mathrm{~m}$ in length, held up by a stand, with the endpoints, 0 and 100 marked. The relatively large number line length was used to decrease the difficulty in differentiating the spatial location of close number values. Participants were shown a symbolic number that was held up by the experimenter who stood behind the number line. Participants were asked to place a physical marker corresponding to its position on the number line. Participants manually placed a small marker on the $1 \mathrm{~m}$ length line (the market stuck to the line via Velcro). Stimuli were presented in a randomized order (see Appendix). Performance on this task was calculated as the mean deviation between where participants placed the marker and the actual number location. For example, if the participant placed the marker for 50 where 65 actually then the error for that trial was 15.

\section{Results}

\section{Non-symbolic number comparison task}

We evaluated if learners' performance depended on ratio difference, absolute value, or the interaction of ratio difference and absolute value. Though there is initial evidence of the effect of absolute value on numerical comparison with adults it is unclear if such an effect would be present with children (Prather, 2014).

Overall participants' performance on the numerical comparison task was $60 \%$ correct (see Figure 3). We evaluated participants' performance on the numerical comparison task using a series of logistic regression analyses. In each analysis we evaluated which set of factors best predicts trial correctness. We used the following variables: the trial ratio difference (log transformed and standardized), trial absolute value (standardized) and their interaction. Participant age strongly correlated with overall performance $(R=0.48, p<0.01)$. The focus of this study is on which factors account for variation in participants' behavior when controlling for age. Thus all regression analyses included participant age and identity as random factors. This allows us to "control for" participant age and individual variation in performance. Each regression analysis includes 51 data points per subject.

Regression analysis 1 included only ratio difference as a predictor of trial correctness. Ratio difference was a significant predictor $(B=0.13, z=3.18, p=0.001)$. As expected larger ratio differences were associated with increased likelihood of a correct response. 
Regression analysis 2 included ratio difference and absolute value as predictors of trial correctness. Ratio difference $(B=0.13, z=3.19, p=0.001)$ was a significant predictor while absolute value $(B=-0.01, z=0.29, p=0.76)$ was not. A comparison of regression analyses indicated that analysis 2 did not account for more variance than analysis $1, X^{2}=0.08, p=0.77$.

Regression analysis 3 included ratio difference, absolute value and their interaction as predictors. Ratio difference $(B=0.13, z=3.05, p<0.001)$, and the interaction of ratio difference and absolute value $(B=-0.11, z=2.60, p=0.01)$ were significant predictors of trial correctness. Absolute value was not a significant predictor $(B=-0.01, z=-0.025, p=0.79)$.

A comparison of the three regression models indicated that regression model 3 accounted for more variance than both analysis $1, X^{2}=6.85, \mathrm{p}=0.03$ and analysis $2, X^{2}=6.76, \mathrm{p}<0.01$. Thus, participants' performance is best described by the regression analysis that includes ratio difference, absolute value, and their interaction as significant predictors. This result replicates recent work with adult participants that numerical comparison difficulty is predicted by both ratio difference and the interaction of absolute value and ratio difference (Prather, 2014). The interaction of ratio difference and absolute value indicates that absolute value effects likelihood for correct response more so for smaller ratio values.

\section{Number Line task}

We evaluated participants' performance on the number line task by measuring the deviation from where the participant placed a value and its actual location. If a participant placed the value 20 at the location for 51, the error on that trial was 31 . We use raw differences, and not ratio differences because ratio differences biases the results towards the performance with smaller numbers. Overall the mean error across all participants was $\mathrm{M}=14.15 \mathrm{SD}=7.81$ (maximum $=39.27$, minimum $=3.3$ ). Participant performance was highly correlated with age $(\mathrm{R}=$ $0.59, \mathrm{p}<0.01)$ Participants' overall performance was better fit by a linear best-fit line $(\mathrm{R}=0.96)$ than logarithmic $(\mathrm{R}=0.92)$ or two-cycle power model $(\mathrm{R}=0.91)$ and best fit by a "s curve" fit using cubic equation with 2 free parameters at $\mathrm{R}=0.98$ (see Barth \& Paladino, 2011).

\section{Relation between number line and number comparison}

We examined relation between participant age, comparison task score and number line task score. Older ages were associated with better scores on the comparison task, $\mathrm{R}^{2}=0.247 \mathrm{t}=$ $3.83, \mathrm{p}<0.01$. Older ages were also associated with better number line performance, $\mathrm{R}^{2}=0.348 \mathrm{t}=$ $4.90, \mathrm{p}<0.01$. Lastly, better number line performance, as measured by deviation error, was significantly correlated with better comparison task score, as measured by percent correct, $\mathrm{R}^{2}=$ $0.19 \mathrm{t}=3.28, \mathrm{p}<0.01$.

To test if symbolic number line performance was predicted by number comparison when accounting for age we compared regressions that predicted number line performance based on either age only or age and number comparison performance. Two of the $\mathrm{N}=47$ participants had number line scores that were significant outliers ( $>3$ SD above mean). No other participants were more than 2 SD from the mean. For the remainder of analysis we considered the data without those two participants $(\mathrm{N}=45)$.

For a linear regression predicting number line score with age as a predictor overall fit was $\mathrm{R}=0.58, \mathrm{AIC}=112.65$, and age was a significant predictor, $\mathrm{B}=-0.6, \mathrm{t}=4.91, \mathrm{p}<0.001$. As expected the older the participant the lower their error on the number line task. For a linear regression predicting number line score with age and comparison score as predictors the overall fit was $\mathrm{R}=$ $0.64, \mathrm{AIC}=107.96$. Both age and number comparison were significant predictors, $\mathrm{B}=-0.42 \mathrm{t}=3.23$ 
$\mathrm{p}=0.002$, and $\mathrm{B}=-0.34, \mathrm{t}=2.60 \mathrm{p}=0.013$ respectively. A comparison of variance accounted for by each regression model shows that the model with both variables accounts for significantly more variance, $F(1,42)=6.73, p=0.012$, difference in AIC of 4.69 .

A regression model including the interaction of age and comparison score did not result in a significant interaction $(B=-0.04, t=0.37, p=0.71$ ). Both age and comparison score remained significant predictors individually, $\mathrm{B}=-0.44, \mathrm{t}=3.19, \mathrm{p}=0.002$, and $\mathrm{B}=-0.34, \mathrm{t}=2.53, \mathrm{p}=0.015$ respectively. The lack of interaction suggests no change in the relation between number line and comparison scores across the age range. The regression model with the interaction term did not account for significantly more variance than the two term regression model, $F(1,42)=0.14, p=$ 0.71, difference in AIC of 1.85 .

\section{Experiment 1 Discussion}

The current results suggest that participants' performance on the symbolic number line task is significantly predicted by performance on the number comparison task, even when accounting for age. This is consistent with prior work on correspondence between number line and non-symbolic numerical comparison. We confirm that this relation holds across the age range 5 through 8 years old. Participants' performance on the non-symbolic comparison task was above chance overall though low due to the range of comparisons used.

\section{Experiment 2: Computational Model}

In the following experiment we present a computational model of participants' performance on the numerical comparison and number line estimation tasks. The modeling approach is designed evaluate if variation in neural coding of number can simultaneously account for behavior on both tasks. Prior work has shown that width of the neural tuning curves can be used to predict developmental change in number line estimation (Prather, 2012) and adult performance on numerical comparison (Prather, 2014). Predicting individual differences on both tasks with the same model instantiation is a more stringent assessment of model performance. Thus, the current study is the most comprehensive evaluation of this modeling approach to date.

Learners' behavior on the non-symbolic numerical comparison and number line estimation tasks may rely on the same underlying mechanism. In this experiment we evaluate the possibility that neural coding of number may contribute to individual differences in performance on numerical comparison and number line estimation tasks. The characteristics of the neural coding of numerical perception have been described via both human neuroimaging studies and non-human primate direct recording (e.g., Nieder \& Dehaene, 2009; Nieder \& Merten, 2007). Behavioral errors are associated with neural coding "errors"; on trials with a behavioral error the expected neural activity from the neural populations was reduced (Andreas Nieder \& Merten, 2007). The following set of simulations evaluates if computational models of neural coding can simultaneously account for both numerical comparison and number line estimation performance.

Computational models simulated neural coding associated with numerical stimuli using probabilistic tuning curves that were then applied to both numerical comparison and number line estimation tasks (e.g., Prather, 2012, 2014). The computational models used here simulate these neural tuning curves that correspond to perception of numerosity. Each model instantiation had a performance score for numerical comparison and number line estimation. Performance for numerical comparison was scored as the percept of comparisons where the larger value was correctly selected. Performance for the number line estimation task was the median difference 
between model estimations and the target values. We then compared the variation in performance of the 45 models to that of participants in experiment 1.

\section{Method}

The computational models evaluated the same stimuli as seen by participants in experiment 1 for both tasks. There were 45 independent model instantiations that varied in the coefficient corresponding to the relative width of the neural tuning curves and noise. The coefficients were selected using an evolutionary algorithm to minimize the deviation between model and participant behavior. Thus each participant was pair with a model that was optimized to fit their behavior. We fit each participant-model pair independently and then evaluated how closely the set of model instantiations matched participant behavior.

\section{Modeling specifications}

Simulations were evaluated using MATLAB (Mathworks) software. The computational models were layered neural network models where values within layers represent neural tuning curves. Neural tuning curves have been found in non-human primate research. Tuning curves are the pattern of neural activity that correlates with perception of numerosity. The spiking rate of a given set of neurons is correlated maximally to a particular value $\mathrm{N}$, and less for $\mathrm{N}+1, \mathrm{~N}-1$, etc. (Nieder et al ., 2002; Nieder \& Miller, 2003; Nieder \& Merten, 2007; Sawamura et al ., 2002). This coding creates Gaussian-like neural tuning function. Each number magnitude is not coded exactly, but in a manner that is consistent with Weber-Fechner' s law. Importantly the data also showed that behavioral errors were correlated with neural turning "errors". Neural tuning curves vectors were calculated for number values identical to stimuli in the behavioral experiment. The initial vectors can be interpreted as idealized activation patterns to which some noise is added to determine the model output vectors. The index of the maximum value corresponded to the perceived number value. Variation in spiking rate, e.g. noise, is calculated as a change in the vector values by some percent taken from a random distribution, where the mean noise is zero and standard deviation of 0.25 . The range of noise distribution is equivalent to the coefficient of variation of the tuning curve. The noise range varies with the mean spiking rate and the coefficient of variation (standard deviation/mean) also varies. Model instantiations used a coefficient of variation that increased with firing rate. Neural data with non-human primates shows that the coefficient of variation increases with firing rate (Pearson et al 2010; Roitman et al., 2007). After the application of noise the vector output values were calculated, where the index of the maximum value of the vector equaled the output. The entire process of the application of random noise to the set of tuning functions was repeated 1000 times per model instantiation.

Simulations used vectors to represent spiking rates for neural populations that comprise the neural tuning functions. Each item in the vectors represents the relative activation level, in terms of spiking rate for a population of cortical neurons. Each simulation included one vector for each of the number magnitudes to be estimated. The values in each vector represent the relative activation (spiking rates) of number selective neurons. For example, the numerical value A was represented by the vector $A\left(n_{1}, n_{2}, \ldots n_{250}\right)$, where the value for $n_{x}$ is the spiking rate for the neurons selective for the number magnitude A. For example, the activation value at index 5 corresponds to the mean spiking rate of the neural population that respond maximally to visual display of 5 items. 


$$
f(x)=h e^{\frac{-(x-m)^{2}}{2 s^{2}}}
$$

Activation values for each vector were calculated using a modified Gaussian distribution function that varies in height similar to a Poisson distribution (see above equation). The maximum vale of the tuning curve $h$, varies with the target numerical value $(y)$, such that $h=(121-y)$. The relative width of the calculated curves varied with the value of $s$. The mean of the distribution, $m$ is a constant set to 0 . The distance between the target numerical value $(\mathrm{T})$ and the current vector index (V) is defined as $X=\log _{20} T-\log _{20} V$. The method of defining $X$ by logarithmic differences results in Gaussian functions that are symmetric on a log scale and of identical width. On a linear scale the functions vary in width and positive skew (skew merely refers to the fact that the function is not symmetric about the mean). Smaller values are both narrower and more skewed. Again this is simply the consequence of transforming a Gaussian curve that is symmetric on a log scale to a linear scale. The maximum spiking rate for large numbers is lower than for smaller numbers (e.g. Nieder \& Dehaene, 2009). Similar equations have been used in prior computational work (Dehaene, 2007; Prather, 2012; 2014).

\section{Fitting model to human data}

Model fit was optimized using an evolutionary algorithm. This process is done independently for each participant. Model fitness is calculated using an overall error term that combines the difference between model and participants score on the numerical comparison and number line estimation tasks. The evolutionary algorithm then minimizes this error. Model instantiations were run in batches of 10. For each batch the specifications for noise and turning curve width were randomly selected within a predetermined range. The ten models within the batch were then ranked based on overall error. Models specifications with the lowest error (ranks 1 and 2) were used again in the next batch. Models ranked 3 to 5 were 'mutated' by adjusting the specifications by a small random amount. Models ranked 6 through 10 were discarded. In the next batch newly generated model specifications replaced models previously ranked 6 through 10 . Thus as many batches are run, only model specifications that result in small error are retained.

\section{Results}

\section{Overall Model performance}

We evaluated the model performance in numerical comparison and number line estimation. Model performance on these tasks was calculated similarly to participants in experiment 1; numerical comparison - percent correct, number line estimation - mean error. Model performance on both tasks varied with changes to the tuning curve width and noise parameters. For the number comparison task the 45 models average performance was 58\% correct, with individual model instantiations ranging from $78 \%$ to $41 \%$. For the number line estimation task the models average performance was 20.71 , with individual model instantiations ranging from 6.82 to 52.94 .

\section{Relation between numerical comparison and number line estimation}


We evaluated the relation between model performance on the numerical comparison and number line estimation tasks, in particular as compared to participants in experiment 1. Participants' performance on the two tasks was correlated ( $\mathrm{R}=0.55, \mathrm{p}<0.01$ ) (See Figure 4). Model instantiations also showed a significant correlation in performance between the two tasks

$(\mathrm{R}=0.55, \mathrm{p}<0.01)$. The association between number comparison and number line is the same for the model and behavioral data. Standard deviation for the comparison task was 0.08 for participants and 0.05 for the model. For the number line task participant standard deviation was 5.91 and model data 10.46 .

\section{Comparison between model and human data}

To compare model and human data directly we matched each model instantiation with its human participant pair from the evolutionary algorithm. We compared model and human data using paired T-tests. For the numerical comparison task model and human performance were significantly different, $\mathrm{t}(2.16), \mathrm{p}=0.03$. For the number line estimation task model and human performance were significantly different, $t(8.61), p<0.01$. The model-participant correlation for the comparison task was $R=0.65(p<0.01)$ and for the number line task $R=0.87(p<0.01)$. The number line task correlation was significantly higher than the comparison task correlation, $\mathrm{z}=$ $2.56, \mathrm{p}=0.01$. Thus the model data fit participants' number line data much closer than numerical comparison data.

\section{Experiment 2 Discussion}

We interpret the computational modeling results as evidence the neural tuning curve model can only partially account variability in participants' behavior across numerical comparison and number line estimation tasks simultaneously. The model fit to behavioral data produces significant correlations for both tasks. However, the model data deviation from behavioral data is statistically significant. Results do not preclude the existence of other factors that may also contribute to the correlation. Model results provide evidence that variation in neural coding of number can be used to predict individual differences across multiple numerical tasks. That neural coding can simultaneously account for individual differences on multiple numerical tasks suggests that it is an effective mechanistic account of numerical cognition.

Recall that the model assumes neural tuning curves can be used as a measure of numerical representation and that the relative width of the tuning curves corresponds to the precision of the numerical representation. The neural tuning curve modeling approach has predicted developmental change on the number line estimation task (Prather, 2012) and has also predicted the effect of absolute value on numerical comparison task performance (Prather, 2014). We attempt, in this study to extend the neural tuning curve modeling approach to predicting individual participants performance on both number line and numerical comparison tasks simultaneously. The success of the model in predicting both tasks simultaneously is mixed. In some sense the model performs well, correlations between model and human data are high for both tasks ( $\mathrm{R}=0.65$, and $\mathrm{R}=0.87)$. However, for both tasks the model performance was statistically different from the human data. We interpret these results, as evidence that the neural tuning curve model can account for a significant amount of variation on the two tasks does not eliminate the possibility of other major factors. 


\section{General Discussion}

The focus of the current study was to evaluate if a neural tuning curve computational model could account for the relation between participants numerical comparison and number line estimation performance. The results demonstrate that children's number line performance can be predicted by non-symbolic numerical comparison while accounting for age. Computational modeling results demonstrate how modeling neural tuning curves can be used to predict individual participant behavior for both tasks. Adjusting the width of modeled tuning curves can capture the individual differences in participant behavior. Though the results show large correlations between model and participant data for both tasks the fit is not precise. The computational data show the same trend as human data with less variability, as illustrated by the difference in standard deviations for modeling and human data. For example, in the human data multiple participants with nearly identical number line estimation scores had a range of different numerical comparison scores. The model results suggest an additional source of variation in participants' performance is necessary to account for the relation between the number line and numerical comparison tasks. The number line apparatus used in this study was longer than some of the various lengths used in prior work. It is possible that the current results show more of an influence of participants' spatial mapping ability and motor control than prior work.

We have shown that the neural coding computational model of number comparison and number line estimation fails to fully account for individual behavior. It is possible that variation due to proportional reasoning skill or other cognitive skills such as inhibitory control may fill that gap (Barth \& Paladino, 2011; Gilmore et al., 2013; Sullivan, Juhasz, Slattery, \& Barth, 2011). If so this would be consistent with the idea that children's performance on the number line task is not due to their "mental number line" or other number specific representations. For example there may be some variance in participants' spatial mapping ability that is not specific to number representation (Barth \& Paladino, 2011). The contribution of proportional reasoning, inhibition and neural coding of number to participants' behavior on the number line task are not mutually exclusive. Further research is required to determine the exact nature of these contributions, as the current data does not address this specifically.

One larger goal of research in numerical cognition is to describe what underlying representations learners use to perform numerical tasks. The current results demonstrate that computational model on neural coding is useful in predicting individual differences across numerical comparison and number line estimation tasks. We demonstrate that models of neural tuning curves can be fit to individual participants' performance using only variation in neural tuning curve width. Learners' behavior has been shown to vary with even small changes such as the format of numerical presentation. This variation in behavior presents a problem and opportunity for researchers. This makes it difficult to pin down a particular mental representation that totally accounts for the observed behavior. In the current study we evaluate the neural coding representation (Prather, 2012; 2014). Despite prior success using this representation to predict symbolic number line performance, and non-symbolic comparison we do not find evidence of predicting individual differences across both tasks well. This lack of success should be interpreted as neural coding representations as useful, in describing behavior but not sufficient. Given the variation in behavior on numerical tasks it is not surprising that a simple explanation fall short. It may be the case that a comprehensive understanding of numerical cognition depends on multifaceted representations that may include neural coding. How these different representations relate to each other and are invoked by the learner remains an outstanding question. 
Future work will focus on how can mechanisms be integrated into computational models of behavioral relevant to numerical tasks. The first goal is to more precisely predict individual differences on numerical comparison, number-line estimation and other numerical tasks. Secondly we will work to expand to set of numerical tasks than can be simultaneously integrated into the modeling approach. The overall end goal is a precise and accurate predictive model of children's performance on wide range of numerical and mathematical tasks.

\section{References}

Barth, H. C., \& Paladino, A. M. (2011). The development of numerical estimation: evidence against a representational shift. Developmental Science, 14(1), 125-35.

http://doi.org/10.1111/j.1467-7687.2010.00962.x

Beddington, J., Cooper, C. L., Goswami, U., Huppert, F. A., Jenkins, R., Jones, H. S., ... Thomas, M. (2008). The mental wealth of nations. Nature, 455(October), 1057-1059.

Cohen, D. J., \& Blanc-Goldhammer, D. (2011). Numerical bias in bounded and unbounded number line tasks. Psychonomic Bulletin \& Review, 18(2), 331-8. http://doi.org/10.3758/s13423-0110059-z

Cohen, D. J., \& Sarnecka, B. W. (2014). Children's number-line estimation shows development of measurement skills (not number representations). Developmental Psychology, 50(6), 16401652. http://doi.org/10.1037/a0035901

Cragg, L., \& Gilmore, C. (2014). Skills underlying mathematics: The role of executive function in the development of mathematics proficiency. Trends in Neuroscience and Education, 3, 63-68. http://doi.org/10.1016/j.tine.2013.12.001

Dehaene, S. (2007). Symbols and quantities in parietal cortex : elements of a mathematical theory of number representation and manipulation. In Sensorimotor foundations of higher cognition (pp. 527-574).

Fazio, L. K., Bailey, D. H., Thompson, C. a, \& Siegler, R. S. (2014). Relations of different types of numerical magnitude representations to each other and to mathematics achievement. Journal of Experimental Child Psychology, 123, 53-72. http://doi.org/10.1016/j.jecp.2014.01.013

Geary, D. C., Hoard, M. K., Nugent, L., \& Byrd-Craven, J. (2008). Development of number line representations in children with mathematical learning disability. Developmental Neuropsychology, 33(3), 277-99. http://doi.org/10.1080/87565640801982361

Gilmore, C., Attridge, N., Clayton, S., Cragg, L., Johnson, S., Marlow, N., ... Inglis, M. (2013). Individual Differences in Inhibitory Control, Not Non-Verbal Number Acuity, Correlate with Mathematics Achievement. PLoS ONE, 8(6), e67374. http://doi.org/10.1371/journal.pone.0067374 
Gunderson, E. a, Ramirez, G., Beilock, S. L., \& Levine, S. C. (2012). The relation between spatial skill and early number knowledge: the role of the linear number line. Developmental Psychology, 48(5), 1229-41. http://doi.org/10.1037/a0027433

Halberda, J., \& Feigenson, L. (2008). Developmental change in the acuity of the "Number Sense": The Approximate Number System in 3-, 4-, 5-, and 6-year-olds and adults. Developmental Psychology, 44(5), 1457-65. http://doi.org/10.1037/a0012682

Halberda, J., Mazzocco, M. M. M., \& Feigenson, L. (2008). Individual differences in non-verbal number acuity correlate with maths achievement. Nature, 455(7213), 665-8. http://doi.org/10.1038/nature07246

Holloway, I. D., \& Ansari, D. (2008). Domain-specific and domain-general changes in children's development of number comparison. Developmental Science, 11(5), 644-9. http://doi.org/10.1111/j.1467-7687.2008.00712.x

Izard, V., \& Dehaene, S. (2008). Calibrating the mental number line. Cognition, 106(3), 1221-47. http://doi.org/10.1016/j.cognition.2007.06.004

Landy, D., Charlesworth, A., \& Ottmar, E. (2016). Categories of Large Numbers in Line Estimation. Cognitive Science, 41(2), 326-353. http://doi.org/10.1111/cogs.12342

Laski, E. V, \& Siegler, R. S. (2007). Is 27 a big number? Correlational and causal connections among numerical categorization, number line estimation, and numerical magnitude comparison. Child Development, 78(6), 1723-43. http://doi.org/10.1111/j.1467-8624.2007.01087.x

Libertus, M. E., Odic, D., \& Halberda, J. (2012). Intuitive sense of number correlates with math scores on college-entrance examination. Acta Psychologica, 141(3), 373-379. http://doi.org/10.1016/j.actpsy.2012.09.009

Marr. (1982). Vision: A Computational Approach.

Nieder, A. (2005). Counting on neurons: The neurobiology of numerical competence. Nature Reviews Neuroscience, 6, 177-190.

Nieder, A. (2013). Coding of abstract quantity by "number neurons" of the primate brain. Journal of Comparative Physiology. A, Neuroethology, Sensory, Neural, and Behavioral Physiology, 199(1), 1-16. http://doi.org/10.1007/s00359-012-0763-9

Nieder, A., \& Dehaene, S. (2009). Representation of number in the brain. Annual Review of Neuroscience, 32, 185-208. http://doi.org/10.1146/annurev.neuro.051508.135550

Nieder, A., \& Merten, K. (2007). A labeled-line code for small and large numerosities in the monkey prefrontal cortex. The Journal of Neuroscience, 27(22), 5986-93.

http://doi.org/10.1523/JNEUROSCI.1056-07.2007 
Opfer, J. E., \& Siegler, R. S. (2007). Representational change and children's numerical estimation. Cognitive Psychology, 55(3), 169-95. http://doi.org/10.1016/j.cogpsych.2006.09.002

Parsons, S., \& Bynner, J. (1997a). Education + Training Emerald Article : Numeracy and employment Numeracy and. Education + Training, 39(2), 43-51.

Parsons, S., \& Bynner, J. (1997b). Numeracy and employment. Education + Training, 39(2), 43 - 51.

Prather, R. W. (2012). Connecting neural coding to number cognition: a computational account. Developmental Science, 15(4), 589-600. http://doi.org/10.1111/j.1467-7687.2012.01156.x

Prather, R. W. (2014). Numerical discrimination is mediated by neural coding variation. Cognition, 133(3), 601-610. http://doi.org/10.1016/j.cognition.2014.08.003

Reid, E. E., Baroody, A. J., \& Purpura, D. J. (2015). Assessing Young Children's Number Magnitude Representation: A Comparison Between Novel and Conventional Tasks. Journal of Cognition and Development, 16(5), 759-779. http://doi.org/10.1080/15248372.2014.920844

Roitman, J. D., Brannon, E. M., \& Platt, M. L. (2007). Monotonic Coding of Numerosity in Macaque Lateral Intraparietal Area. PLoS Biology, 5(8), 1672-1682.

http://doi.org/10.1371/journal.pbio.0050208

Sasanguie, D., Göbel, S. M., Moll, K., Smets, K., \& Reynvoet, B. (2013). Approximate number sense, symbolic number processing, or number-space mappings: what underlies mathematics achievement? Journal of Experimental Child Psychology, 114(3), 418-31. http://doi.org/10.1016/j.jecp.2012.10.012

Sasanguie, D., \& Reynvoet, B. (2013). Number Comparison and Number Line Estimation Rely on Different Mechanisms. Psychologica Belgica, 53(4), 17-35.

Schneider, M., Beeres, K., Coban, L., Merz, S., Schmidt, S. S., Stricker, J., \& Smedt, B. De. (2016). Associations of non-symbolic and symbolic numerical magnitude processing with mathematical competence : a meta-analysis. Developmental Science, 1-16. http://doi.org/10.1111/desc.12372

Sella, F., Berteletti, I., Lucangeli, D., \& Zorzi, M. (2015). Varieties of quantity estimation in children. Developmental Psychology, 51(6), 758-770. http://doi.org/10.1037/a0039183

Siegler, R. S., \& Opfer, J. E. (2003). The development of numerical estimation. Psychological Science, 237-243.

Slusser, E., \& Barth, H. (2017). Intuitive proportion judgement in number-line estimation.

Sullivan, J. L., Juhasz, B. J., Slattery, T. J., \& Barth, H. C. (2011). Adults' number-line estimation strategies: Evidence from eye movements. Psychonomic Bulletin \& Review, 18(3), 557-63. http://doi.org/10.3758/s13423-011-0081-1 


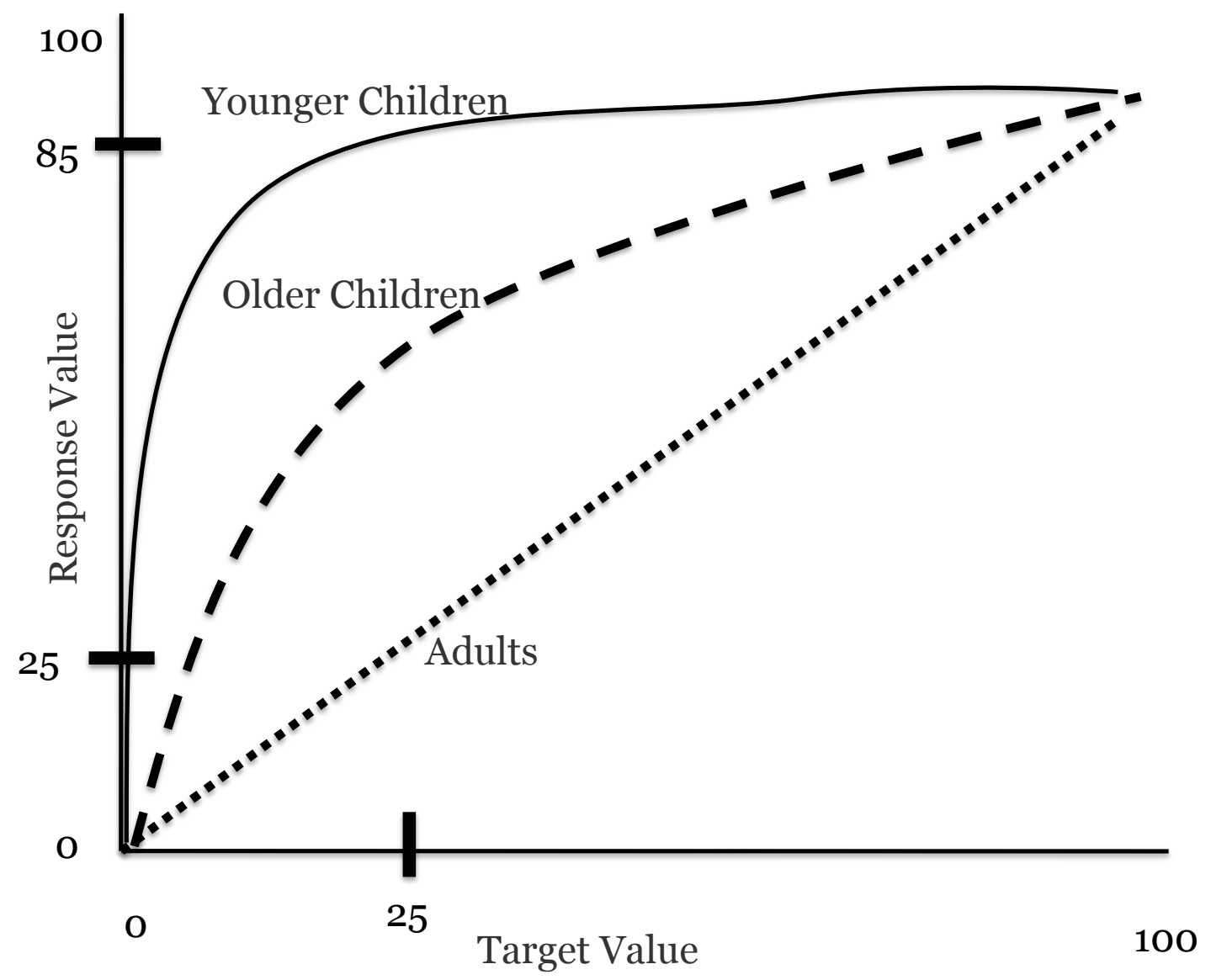

Figure 1. Schematic of typical number line estimation behavior patterns with regard to the logarithmic nature of participants' estimates (for further details see Seigler \& Opfer 2003; Barth \& Paladino 2011). Data is graphed such that the horizontal axis is the value to be placed on the number line, the vertical axis is the location the participant indicated. For example, given the value ' 25 ' adults perform will little error while younger children place the ' 25 ' in the location corresponding to a value of ' 85 '. 


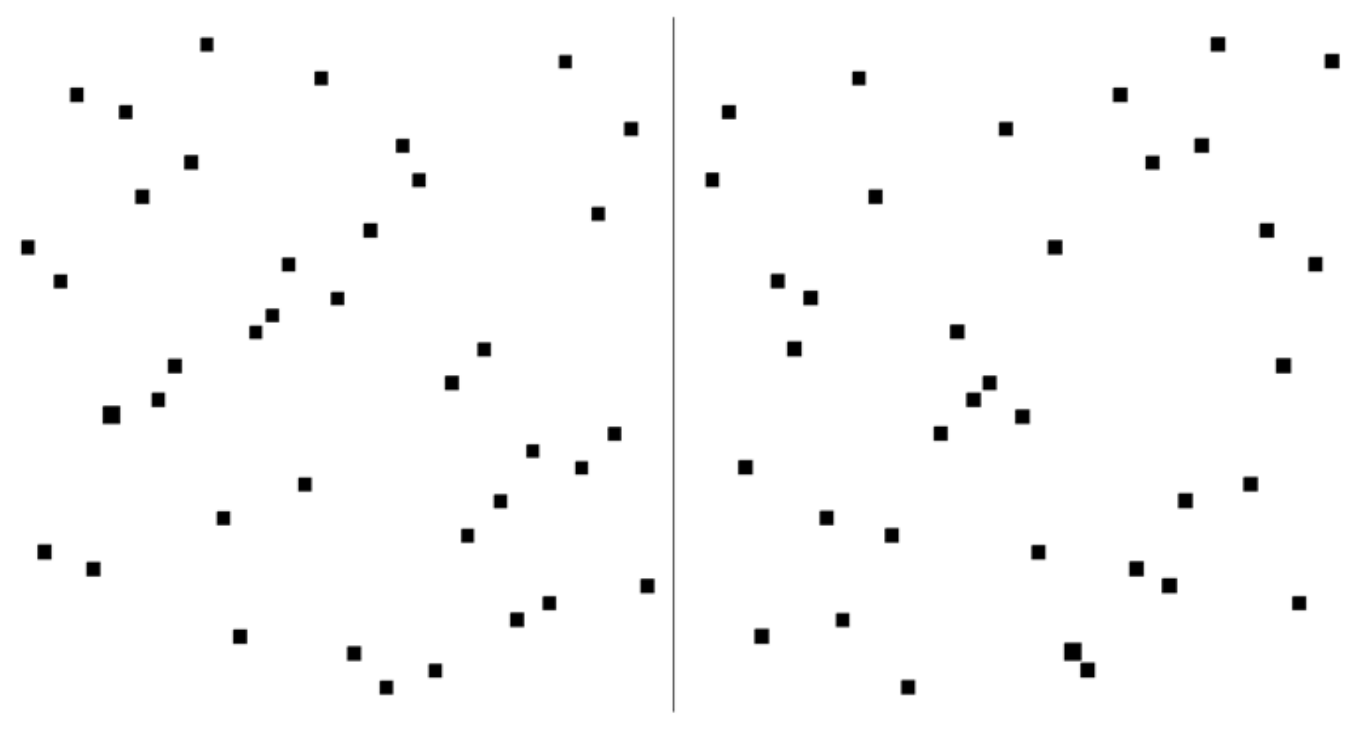

Figure 2. Example of non-symbolic comparison task stimuli. Participants indicate which side of the midline has a larger number of shapes. 


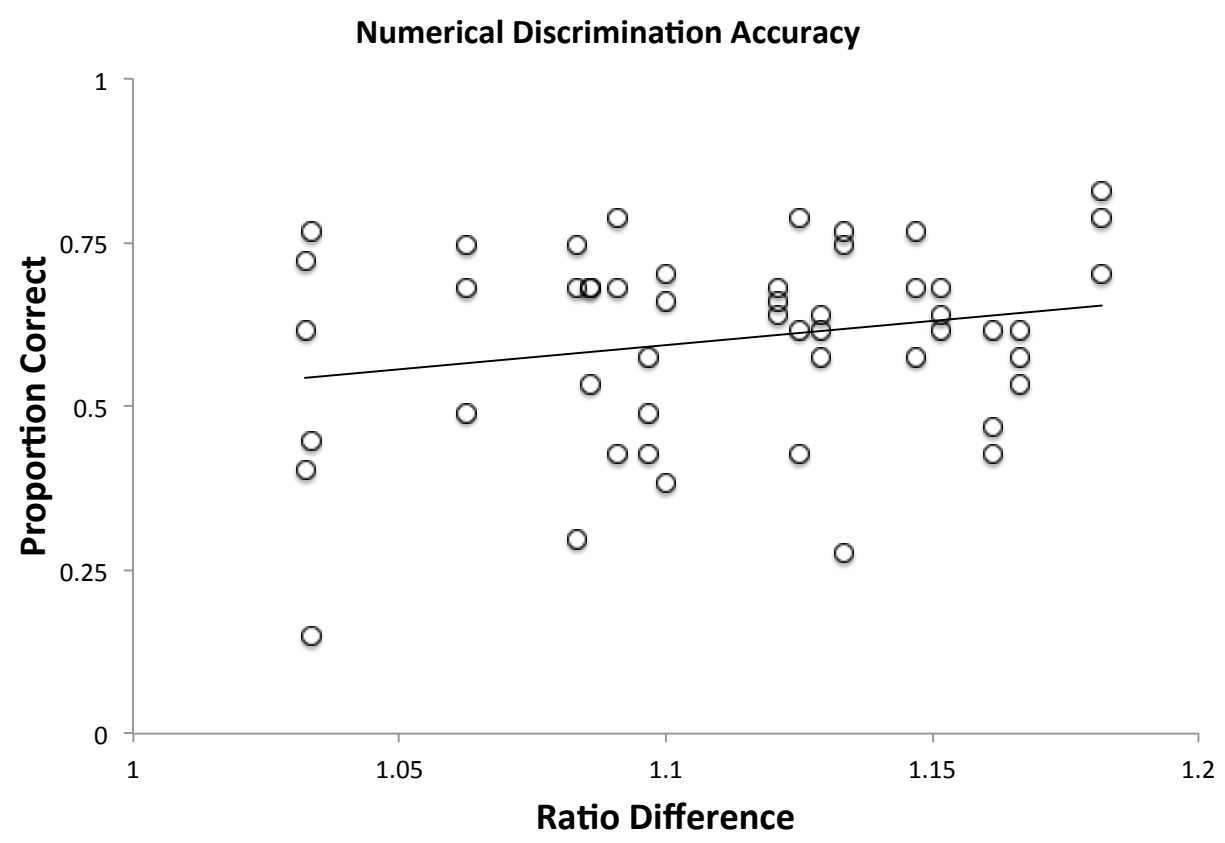

Figure 3. Participants' performance on numerical comparison task. Each point represents one of the 51 different comparisons that all participants completed. The vertical axis is the proportion of participants who correctly judged the comparison (see Appendix for list).

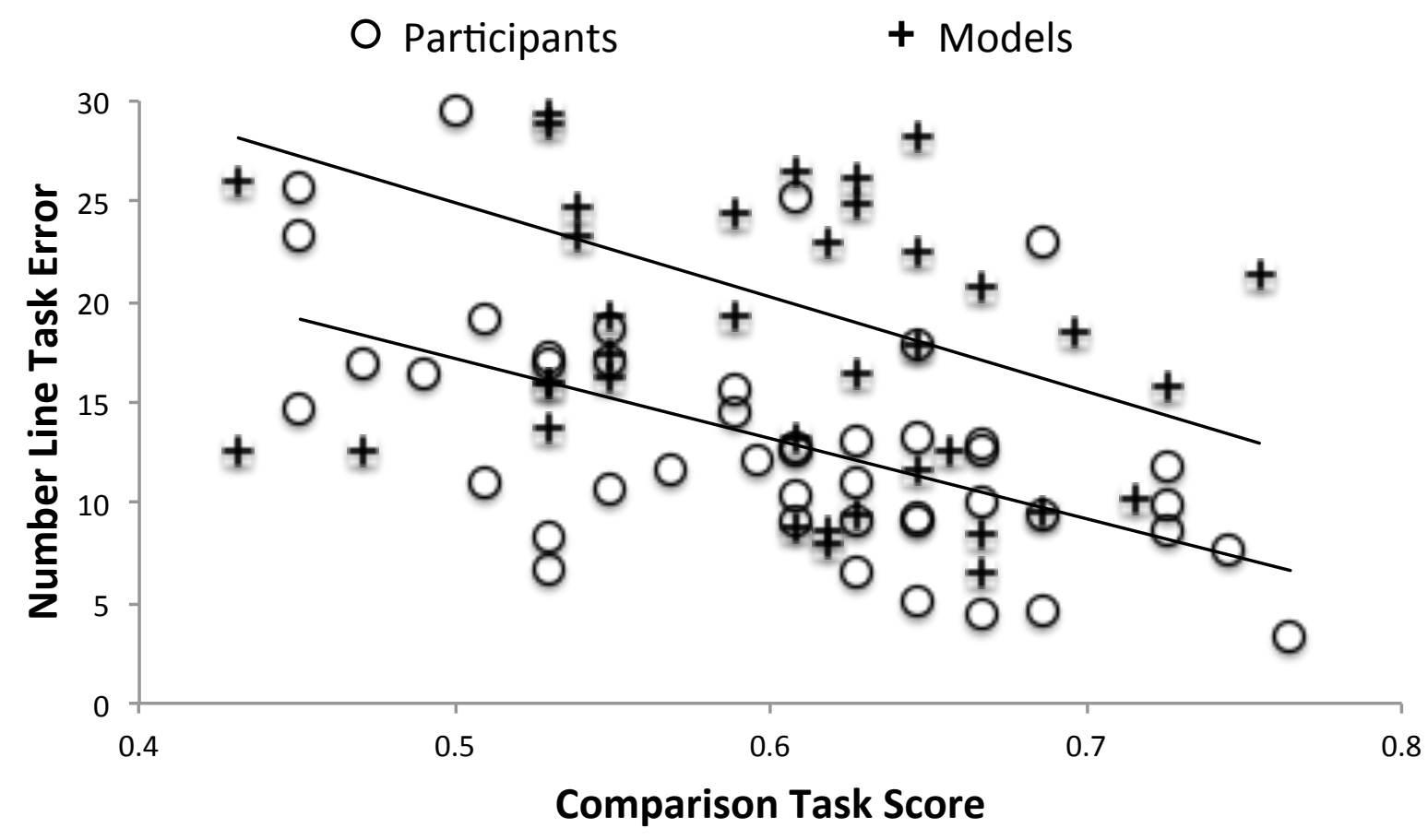


Figure 4. Child and computational model scores for number line estimation and numerical comparison task. Each point represents a single participant or independent instantiation of the computational model. Correlation between model and participant data was $\mathrm{R}=0.93$ 


\section{Appendix}

Non-symbolic Comparison Pairs

$\begin{array}{lllll}32-31 & 78-72 & 102-93 & 35-31 & 76-66 \\ 64-62 & 117-108 & 33-30 & 70-62 & 114-99 \\ 96-93 & 38-35 & 66-60 & 105-93 & 36-31 \\ 31-30 & 76-70 & 99-90 & 34-30 & 72-62 \\ 62-60 & 114-105 & 37-33 & 68-60 & 108-93 \\ 93-90 & 36-33 & 74-66 & 102-90 & 35-30 \\ 34-32 & 72-66 & 111-99 & 39-34 & 70-60 \\ 68-64 & 108-99 & 36-32 & 78-68 & 105-90 \\ 102-96 & 34-31 & 72-64 & 117-102 & 39-33 \\ 39-36 & 68-62 & 108-96 & 38-33 & 78-66 \\ & & & & 117-99\end{array}$

Number Line Task Stimuli

$\begin{array}{lllllll}1 & 4 & 8 & 11 & 14 & 17 & 22 \\ 27 & 30 & 37 & 40 & 45 & 49 & 56 \\ 60 & 68 & 75 & 83 & 92 & 99 & \end{array}$

